\title{
IMPROVEMENT OF STRAIN GAUGES MICRO-FORCES MEASUREMENT USING KALMAN OPTIMAL FILTERING
}

\author{
Y. Haddab, Q. Chen and P. Lutz \\ FEMTO-ST Institute, UMR CNRS 6174 - UFC / ENSMM / UTBM, \\ Automatic Control and Micro-Mechatronic Systems Department \\ 24, rue Alain Savary, \\ 25000 Besançon, France.
}

\begin{abstract}
Manipulation of small components and assembly of Microsystems require force measurement. In the microworld (the world of very small components), signal/noise ratio is very low due to the weak amplitude of the signals. To be used in feedback control or in a micromanipulation system, a force sensor must allow static and dynamic measurements. In this paper, we present a micro-force measurement system based on the use of strain gauges and a Kalman optimal filter. Using a model of the measurement system and a statistical description of the noise, the optimal filter allows filtering the noise without loss of dynamic measurement. The performances of the measurement system are improved and fast force variations can be measured.
\end{abstract}

Keywords: micro-force measurement, Measurement noise, Kalman filter, piezoelectric cantilevers, strain gauges.

\section{INTRODUCTION}

Force measurement is very important to perform manipulation and micro-assembly tasks in the microworld. Small components are often fragile and may be damaged or destroyed if they are grasped without force control. Size reduction makes it difficult to build and integrate very small force sensors. However, in the last decade, various methods have been used in order to measure the force applied on the manipulated objects. Generally, micro-forces are evaluated from a deformation measurement of a small device (cantilever, membrane, etc.) whose stiffness is known. This deformation is measured using a position sensor.

According to the resolution wanted, various effects can be used ( $\mathrm{Lu}$ et al., 2006). For measurements below $0.1 \mu \mathrm{N}$, piezoelectric materials, in particular PVDF (Polyvinylidene Fluoride) are often used. These materials give high resolutions but do not allow static measurements because of the electrical discharge. Fung et al. integrated PVDF sensors into commercial probes in order to characterize MUMPs ${ }^{\circledR}$ (Multi-user MEMS Processes) (Fung et al., 2002). Microfabricated electrostatic sensors are characterized by a good linearity and are able to measure forces as great as $25 \mu \mathrm{N}$ with a resolution as small as $10 \mathrm{nN}$. For better resolutions, optical sensors can be used. AFM (Atomic Force Microscope) cantilevers with laser sensor permit force measurements in the nanometer range. Arai et al, detected forces as small as $3 \mathrm{pN}$ using mechanical characteristics of a calibrated carbon nanotube probe whose deformation is measured by a FE-SEM (Field Emission Scanning Electron Microscope) (Arai et al., 2003). Small strain gauges are also very often used. They can be glued on the device and their performances depend on the characteristics of the deformable device. Cantilevers with a length in the centimeter range allow resolutions in the $\mathrm{mN}$ range and microfabricated cantilevers can reach $\mu \mathrm{N}$ resolutions.

To be used in feedback control or in a micromanipulation system, a force sensor must allow static and dynamic measurements. PVDF materials are not suitable for static measurements. Capacitive sensors are very sensitive but give limited stroke. Optical sensors are often external sensors and are not adapted for embedded applications or confined environments. Strain gauges constitute low-cost easily integrated sensors and represent good candidates for multi-purposes micro-forces measurement.

A general approach consists in gluing one or more gauges on a cantilever close to the fixed end for best sensitivity. When a force is applied at the tip, the deformation induces a resistance change in the gauges. 
Kemper designed a microgripper equipped with a strain gauge (Kemper, 2004). The force measurement resolution is $2 \mathrm{mN}$. Nasir et al. developed a multiDOF microfabricated force sensor based on the piezoresistive effect in order to measure insect flight forces (Nasir et al., 2006). The sensor has a $\mu \mathrm{N}$ range resolution. Woods et al. used piezoresistive gauges to measure flight force of a micromechanical flying insect (Woods et al., 2001). Dao et al. designed, fabricated and calibrated a 6-degree of freedom force-moment micro sensing chip utilizing the piezoresistance effect in silicon (Dao et al., 2003). Deok-Ho Kim et al. implemented a piezoresistive MEMS cantilever for force measurement in micro/nano robotic applications. For a limited stroke, the resolution obtained is better than $1 \mathrm{nN}$ (Kim et al., 2004).

Many other papers reported successful implementation of strain gauges for force measurement. However, the performances presented are often based on a theoretical resolution combining a high gain amplifier, a high resolution ADC (Analog to Digital Converter) and a very-low pass filter. In real microsystems, signal/noise ratio is very low due to the weak amplitudes of the signals for high resolution measurements. Low-pass filtering allows high resolution measurements of static and slowly varying forces but fast forces are filtered. This is a severe limitation for the use in high performance controlled micromanipulators.

In this paper, we present a micro-force measurement system based on the use of strain gauges and a Kalman optimal filter. Using a model of the measurement system and a statistical description of the noise, the optimal filter allows filtering the noise without loss of dynamic measurement. The performances of the measurement system are improved and fast force variations can be measured. This measurement system is well adapted for critical applications where dynamic error must be low.

First, the designed system is described. Second, both static and dynamic characterizations are performed and a model is built. After that, Force measurement results using strain gauges are presented. A Kalman optimal filter is then implemented and measurement results are given. Finally the performances of the optimal filter are discussed.

\section{DESCRIPTION OF THE SYSTEM}

The system designed for this study is used to perform micromanipulation tasks. It is made up of two parts (see figure 1). The first one is a linear motor from PiezoMotor ${ }^{\circledR}$. This motor uses piezoelectric actuators in stick-slip mode and is characterized by a resolution of $10 \mathrm{~nm}$ and a stroke of $35 \mathrm{~mm}$. The second one is a piezoelectric bimorph cantilever equipped with electrodes. The dimensions of this cantilever are: $16 \mathrm{~mm} \times 2 \mathrm{~mm} \times 0.5 \mathrm{~mm}$. When a voltage is applied across the electrodes, the cantilever bends.

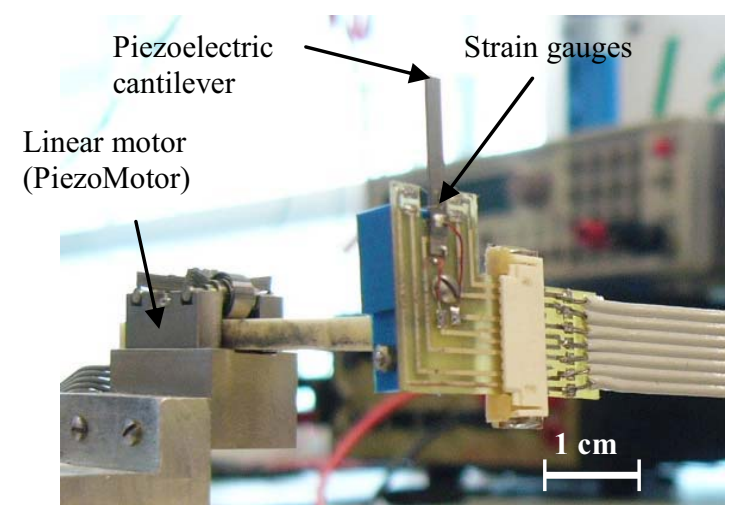

Fig. 1. Constitution of the designed system.

The whole system constitute a half of a gripper and the tip of the cantilever is used to handle a microobject.

Two small strain gauges from ENTRAN ${ }^{\circledR}$ are glued on the two faces of the cantilever close to the fixed end (see figure 2). They are used for force measurement. The dimensions of the gauges are: $1.27 \mathrm{~mm} \times 0.38 \mathrm{~mm}$.

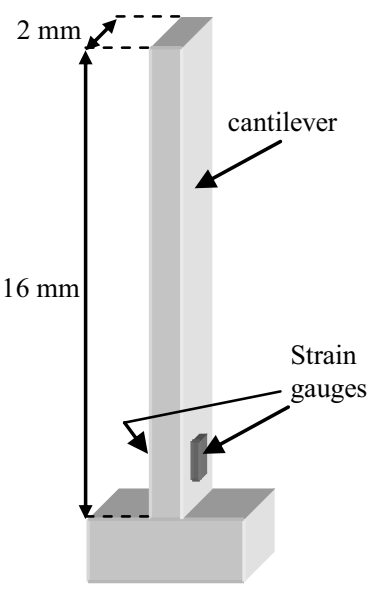

Fig. 2. Bending cantilever equipped with strain gauges.

\section{IDENTIFICATION OF THE SYSTEM}

The designed system allows us to use both parts as actuator: the linear motor for high stroke/high resolution displacements and the piezoelectric cantilever to perform fast displacements. However in our study, we use the cantilever equipped with the strain gauges only as a force sensor. Moreover we will not study the control of the linear motor.

When a force is applied at the tip of the cantilever, it bends causing the deformation of the gauges. Assuming small deformations and a calibration, the measurement of the displacement of the tip allow us to measure the applied force.

\subsection{Static Characterization}

First, a calibration of the cantilever is done. Calibrated weights are used to apply forces at the tip 
of the cantilever and the displacement is measured by a high resolution $(10 \mathrm{~nm})$ laser sensor from KEYENCE®. The results show a good linearity and a compliance of $1.23 \mu \mathrm{m} / \mathrm{mN}$.

\subsection{Dynamic Characterization}

Static force measurement is useful to evaluate the force applied by the gripper or the compliance of a micro-object. However dynamic force measurement is required to perform dexterous manipulation of fragile micro-objects.

The piezoelectric cantilever can be considered as a two inputs-one output system (see figure 3 ). The two inputs are the force $\mathrm{F}$ applied at the tip and the voltage $\mathrm{V}$ applied on the electrodes and the output is the deflection $\delta$.

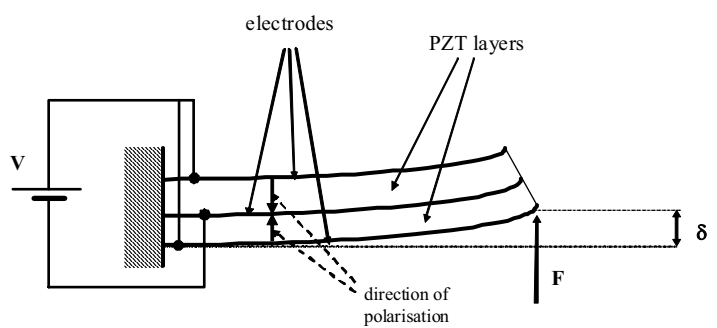

Fig. 3. Input/output signals of the piezoelectric cantilever.

For small displacements, the behavior of the cantilever can be considered as linear. It has been shown (Haddab et al., 2000) that the dynamic relationship between $\mathrm{F}$ and $\delta$ and between $\mathrm{V}$ and $\delta$ are similar.

The three signals are linked by the following equation:

$$
\delta(s)=k_{1} G(s) F(s)+k_{2} G(s) V(s)
$$

where:

$\mathrm{S}$ is the Laplace variable,

$F(s)$ and $V(s)$ are the Laplace transforms of $F(t)$ and $\mathrm{V}(\mathrm{t})$,

$\mathrm{G}(\mathrm{s})$ is a transfer function describing the dynamic behavior of the cantilever,

$\mathrm{k} 1$ and $\mathrm{k} 2$ are the static gains between $\mathrm{F}$ and $\delta$, and $\mathrm{V}$ and $\delta$ respectively.

From the previous static characterization, we can deduce that:

$$
k_{1}=1.23 \times 10^{-3} \mathrm{~m} / \mathrm{N}
$$

The gain $\mathrm{k} 2$ can be easily identified by applying voltages and measuring the deflection using the laser sensor.

$$
k_{2}=9.17 \times 10^{-7} \mathrm{~m} / \mathrm{V}
$$

In order to determine $\mathrm{G}(\mathrm{s})$, a voltage step is applied and the step response is recorded using the laser sensor (see figure 4).

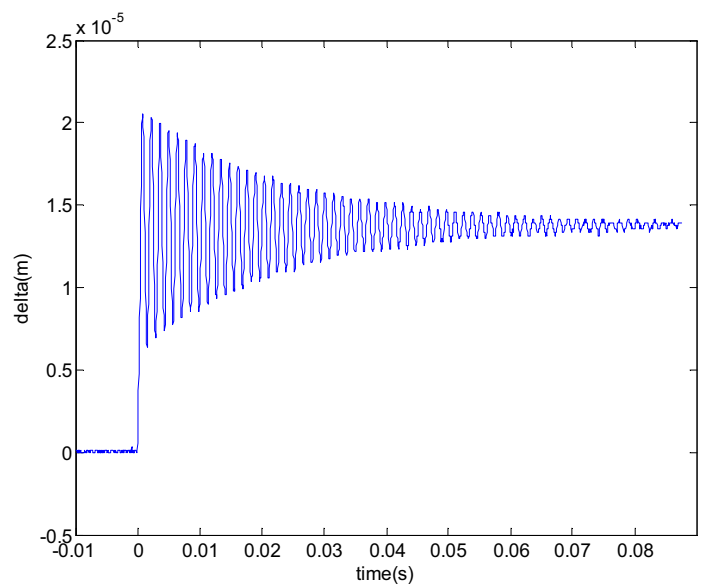

Fig. 4. Step response of the cantilever according to a voltage step of $15 \mathrm{~V}$.

Using ARMAX identification algorithm, a discrete transfer function $\mathrm{G}(\mathrm{z})$ is obtained:

$G(z)=\frac{0.03790 z^{3}-0.07201 z^{2}+0.03941 z}{z^{4}-2.75595 z^{3}+2.50860 z^{2}-0.70475 z-0.04258}$

With a sampling period of

$$
T_{s}=4 \times 10^{-5} \mathrm{~s}
$$

\section{FORCE MEASUREMENT USING STRAIN GAUGES}

In order to measure the force, the two strain gauges glued symmetrically on the cantilever are included in a Wheatstone bridge. This configuration results in better thermal compensation and higher signal/noise ratio. In order to obtain a good signal, the standard bonding process (specified by the manufacturer) must be strictly respected. Two other resistors are used to complete the bridge, an amplifier is used to increase the voltage level and the signal is filtered using a low-pass filter (see figure 5). The 4V supply voltage has been chosen according to the characteristics of the gauges. The amplifier has a gain of 51.3 and the cutoff frequency of the filter is $3100 \mathrm{~Hz}$ which is more than four times greater than the resonant frequency of the cantilever.

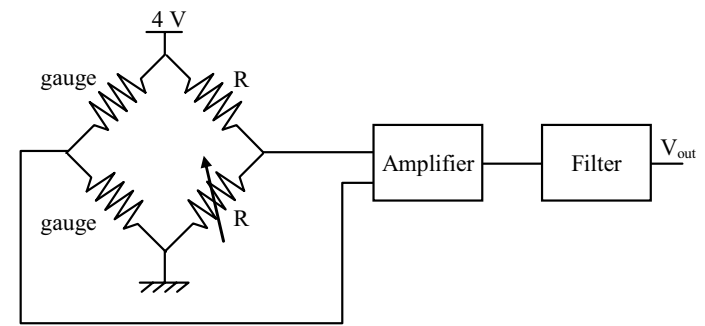

Fig. 5. Setup for force measurement using strain gauges.

As the dynamic behavior is similar for a force input and a voltage input, in order to characterize the 
measurement system, we have chosen to use a voltage step because it is easier to apply. Figure 6 shows the measured voltage in response to a voltage step of $15 \mathrm{~V}$. The measurement system using strain gauges (calibrated using the laser sensor) has a transfer of $52344.6 \mathrm{~V} / \mathrm{m}$. The calibration of the measurement system has been done with the laser sensor.

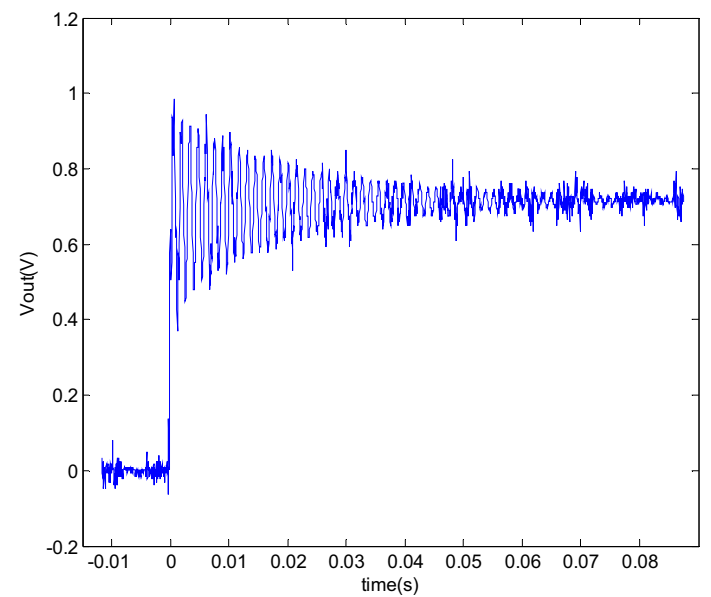

Fig. 6. Measured voltage Vout in response to a voltage step of $15 \mathrm{~V}$.

From the previous step response and the modelization, we notice that the passband of the measurement system is sufficient to reproduce the dynamic behaviour of the cantilever. However, we notice that the level of the measurement noise is high and it limits the measurement's accuracy. Due to the noise, force measurement can be affected by a maximum error of $1.98 \mathrm{mN}$ in the worst case. This noise can be attenuated by reducing the cutoff frequency of the filter but it will result in a loss of dynamic measurement.

\section{KALMAN FILTERING}

In order to filter the measurement noise without loss of dynamic measurement, a discrete Kalman optimal filter is implemented. Several authors have reported successful use of a Kalman filter for force estimation or noise reduction, mainly in macroscopic systems. In (Katupitiya, 1996), a Kalman filter has been used for identification of contact and grasping uncertainties and for monitoring of force controlled assembly operations. (Ma, 2003) and (Liu, 2000) have used a Kalman Filter to estimate forces applied on beam structures. In (Nilsson, 1999) a Kalman Filter is used to help controlling the position of flexible mobile manipulators.

In this section, we present a state space model of the noisy process, the design of the Kalman filter and the obtained results.

\subsection{State space modeling and Kalman filtering}

From the transfer function $\mathrm{G}(\mathrm{z})$, a discrete-time state space model is obtained. Only one input is considered (the voltage $\mathrm{V}$ ).

$$
\begin{aligned}
& X_{k+1}=A \cdot X_{k}+B \cdot V_{k} \\
& \delta_{k}=C \cdot X_{k}
\end{aligned}
$$

Where:

$\mathrm{X}$ is the state vector,

$\mathrm{V}$ is the input voltage,

$\delta$ is the deflection,

$$
\begin{aligned}
A & =\left(\begin{array}{cccc}
0 & 1 & 0 & 0 \\
0 & 0 & 1 & 0 \\
0 & 0 & 0 & 1 \\
0.04258 & 0.70475 & -2.50860 & 2.75595
\end{array}\right) \quad B=\left(\begin{array}{l}
0 \\
0 \\
0 \\
1
\end{array}\right) \\
C & =\left(\begin{array}{llll}
0 & 3.6139 \times 10^{-8} & -6.60332 \times 10^{-8} & 3.47543 \times 10^{-8}
\end{array}\right)
\end{aligned}
$$

If we consider the measurement and the process noises, the system can be represented by the following linear stochastic equation:

$$
\begin{aligned}
& X_{k+1}=A \cdot X_{k}+B \cdot V_{k}+w_{k} \\
& \delta_{k}=C \cdot X_{k}+v_{k}
\end{aligned}
$$

where:

$\mathrm{W}$ and $\mathrm{v}$ represent the process and measurement noises respectively.

Assuming that the noises are independent from each other, white and with normal probability distributions, a discrete Kalman filter provides an efficient recursive method to estimate the state of the process (and therefore the output) in a way that minimizes the mean of the squared error. Figure 7 presents the general structure of the estimation process. $\hat{X}_{k}$ and $\hat{\delta}_{k}$ are the estimated state and output respectively. The algorithm is based on repetition of two steps: time update and measurement update.

In the time update (predict) step, the filter estimates the next state (a priori) of the process according to the current state and error covariance.

In the measurement update (correct) step, the measurement is taken into account in order to obtain an improved state estimate (a posteriori).

More details on the implementation of the Kalman filter are given in (Kalman, 1960) and (Maybeck, 1979).

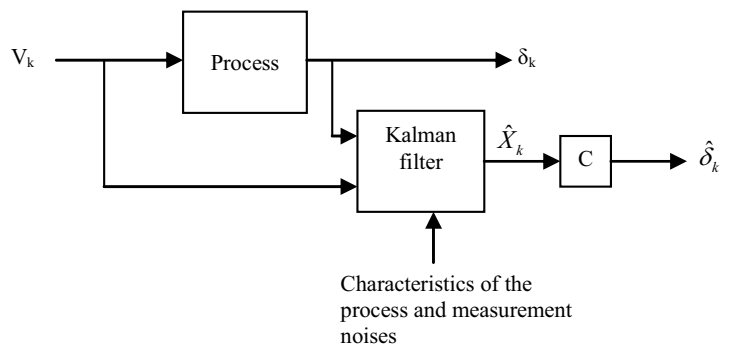

Fig. 7. Estimation of the process state and output using a Kalman filter.

\subsection{Noise characterization}


In order to characterize the noise, we assume that:

- The process noise is mainly due to the input $\mathrm{V}$ (noise produced by the voltage generator),

- The process and measurement noises are independent from each other because they are generated by different devices.

\section{Measurement noise}

The measurement noise is recorded using the strain gauges measurement system when no input is applied (see figure 8).

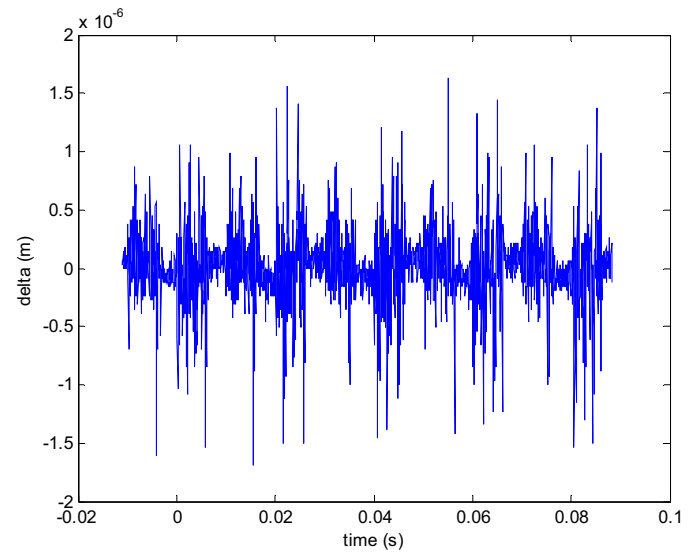

Fig. 8. Measurement noise.

Figure 9 shows that the measurement noise has a Gaussian distribution with a mean of 0 and a variance of $9.02 \times 10^{-14}$.

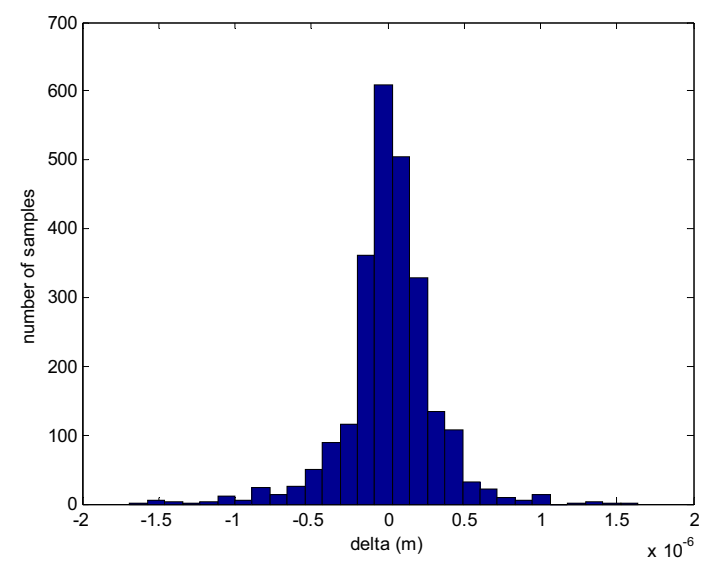

Fig. 9. Distribution of the measurement noise.

The spectrum of the noise, shown in figure 10 , allows us consider the measurement noise as a white noise.

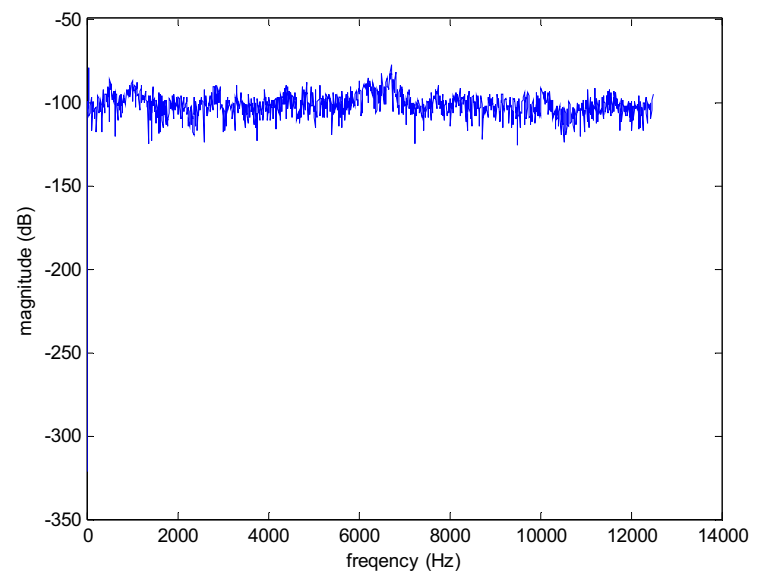

Fig. 10. Spectrum of the measurement noise.

\section{Process noise}

The process noise is assumed to be mainly due to the input noise.

$$
w_{k}=B . v n_{k}
$$

In the previous equation, $v n_{k}$ is the input noise. $v n_{k}$ is measured at the input when a 'zero input' is applied. ('zero input' means that the generator is connected to the system and therefore the noise produced is applied at the input of the system). The same analysis was performed to characterize the input noise. It has shown similar characteristics to those of measurement noise (Gaussian, centered and white) with a variance of $8.14 \times 10^{-9}$.

\subsection{Results}

In this section we present the results of the implementation of the Kalman Filter. In the algorithm, the process noise covariance (covariance of wk) and the measurement noise covariance (covariance of vk) are calculated from noise characteristics.

Figures 11 and 12 show the measured deflection and the estimated deflection using Kalman filtering (respectively) in response to a voltage step of $15 \mathrm{~V}$. These results show that Kalman filtering allow obtaining a significant decrease of measurement noise (see figure 13) without loss of dynamic behaviour measurement (see figure 14).

From figure 13, we can see that the measurement noise amplitude is decreased 40 times and then force measurement can be affected by a maximum error of $50 \mu \mathrm{N}$. 


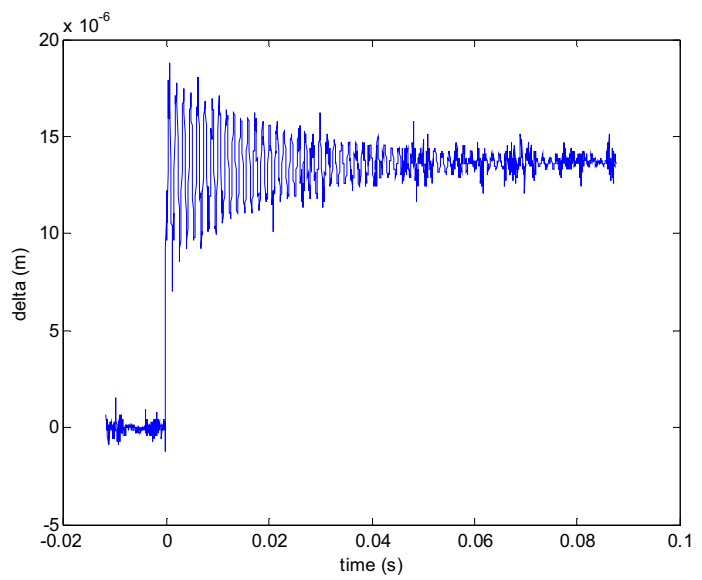

Fig. 11. Measured deflection $\delta$ in response to a voltage step of $15 \mathrm{~V}$.

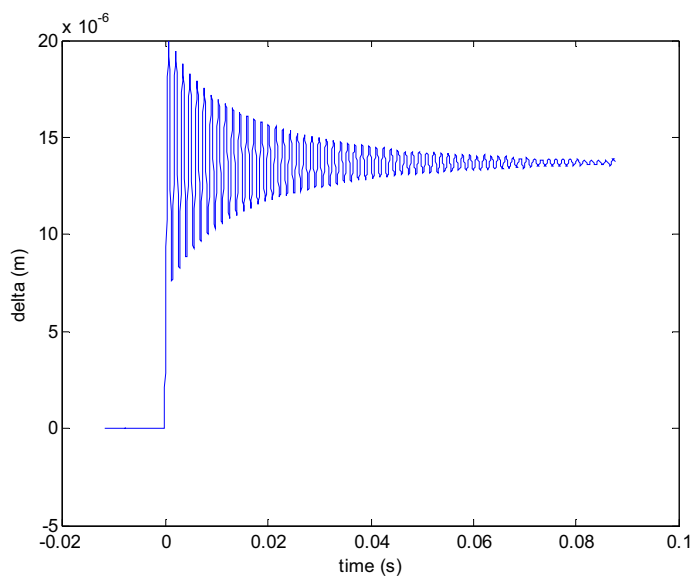

Fig. 12. Estimated deflection $\hat{\delta}$ using Kalman filtering in response to a voltage step of $15 \mathrm{~V}$.

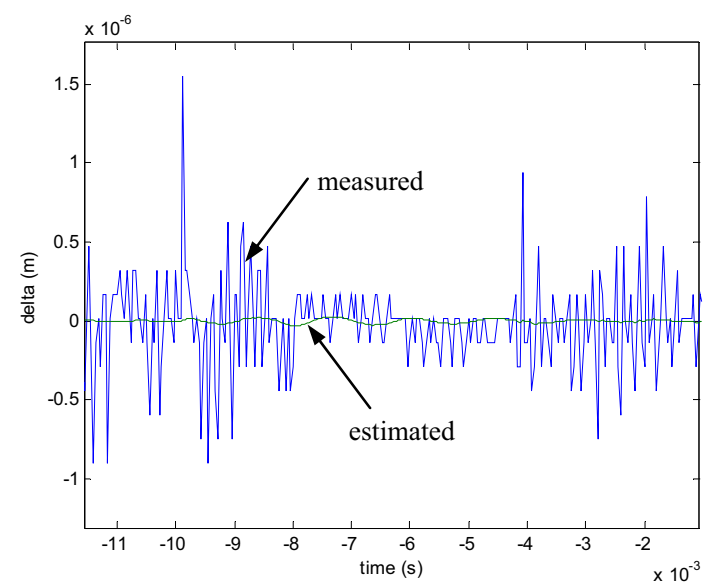

Fig. 13. Comparison between the measured deflection $\delta$ and the estimated deflection $\hat{\delta}$ in static mode.

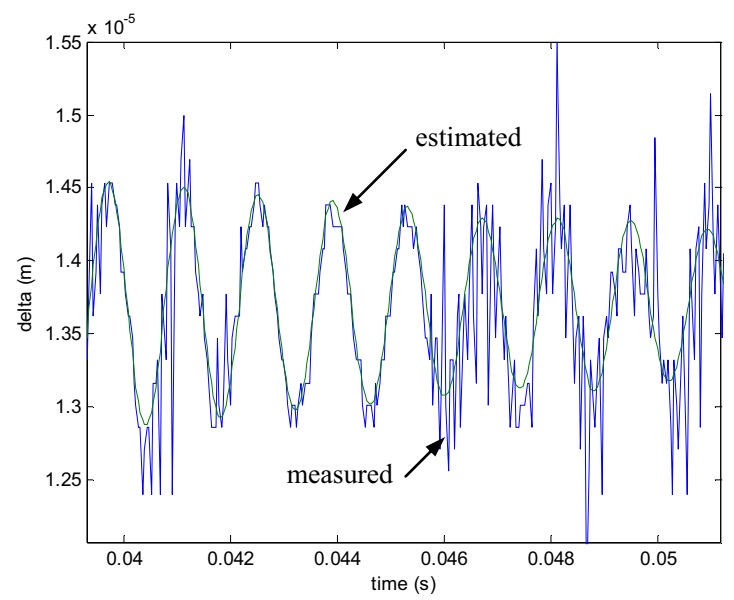

Fig. 14. Comparison between the measured deflection $\delta$ and the estimated deflection $\hat{\delta}$ in dynamic mode.

In the current results, we have considered the voltage input and the noise produced by the voltage generator. Considering the force input will lead to similar approach as the behaviour of the cantilever is similar. However, exact performance of the Kalman filtering will depend on the force noise level. This noise depends on the object in contact with the cantilever tip. In a practical case, the force noise should be evaluated prior to real-time Kalman filtering. Measurement and process noises characterisation can also be periodically updated for best performances. These noises can vary if the working conditions are different or if the gripper is used in different environments.

\section{CONCLUSION AND FUTURE WORK}

Force measurement is very important for the success of manipulation and assembly of small components and devices. In this paper, a measurement system based on the use of strain gauges has been implemented. Strain gauges are low cost, easy to integrate sensors for force measurement.

Because of the weak amplitude of the forces in the microworld, signal/noise ratio is low. Moreover, closed loop control of micromanipulators requires dynamic force measurement. A Kalman optimal filter has been implemented in order to filter the measurement noise and allow measurement of dynamic forces. The use of a Kalman filter allows significant performance improvement.

In the future, the cantilever will be used in a microgripper and the strain gauges and the Kalman filter will be used to estimate the force applied on the manipulated micro-objects. Various manipulation and transportation tasks will be performed in different environmental conditions and the results compared. Moreover, the ability to use the piezoelectric cantilever as actuator and as force sensor opens new perspectives for integrated force/position control. 


\section{REFERENCES}

Arai, F., M. Nakajima, L. Dong and T. Fukuda (2003). The pico-Newton Order Force Measurement with a Calibrated Carbon Nanotube Probe. Proceedings of the 2003 IEEE/ASME International Conference on Advanced Intelligent Mechatronics, pp. 691696, Kobe, Japan.

Dao D. V., T. Toriyama, J. Wells and Susuma Sugiyama (2003). Silicon Piezoresistive SixDegree of Freedom Force-Moment Micro Sensor. Sensors and Materials, vol. 15, $\mathrm{N}^{\circ} 3$, pp. 113-135.

Fung, C. K. M., I. Elhajj, W. J. Li and N. Xi (2002). A 2-D PVDF Force Sensing System for MicroManipulation and Micro-assembly. Proceedings of the 2002 IEEE International Conference on Robotics and Automation, pp. 1489-1494, Washington, DC, USA.

Haddab Y., N. Chaillet and A. Bourjault (2000). A Microgripper Using Smart Piezoelectric Actuators. Proceedings of the 2000 IEEE/RSJ International Conference on Intelligent Robots and Systems. Takamatsu, Japan.

Kalman R. E. (1960). A New Approach to Linear Filtering and Prediction Problems. Transactions of the ASME, Journal of Basic Ingineering, pp. $35-45$.

Katupitiya, J., S. Dutré, S. Demay and J. De Geeter (1996). Estimating Contact and Grasping Uncertainties Using Kalman Filters in Force Controlled Assembly. Proceedings of the 1996 IEEE/RSJ International Conference on Intelligent Robots and Systems, pp. 696-703, Osaka, Japan.

Kemper M. (2004). Development of a Tactile LowCost Microgripper with integrated Force Sensor. Proceedings of the IEEE International Conference on Control Applications, pp. 14611466, Taipei, Taiwan.

Kim D., B. Kim and J. Park (2004). Implementation of a Piezoresistive MEMS Cantilever for Nanoscale Force Measurement in Micro/Nano Robotic Applications. KSME International Journal, Vol. 18, N. 5, pp. 789-797.

Liu, J. J., C. K. Ma, I. C. Kung and D. C. Lin (2000). Input Force Estimation of a Cantilever Plate by Using a System Identification Technique. Computer Methods in Applied Mechanics and Engineering, Elsevier, 190, pp. 1309-1322.

Lu, Z., P. C. Y. Chen and W. Lin (2006). Force Sensing and Control in Micromanipulation. IEEE Trans. On Systems, Man and Cybernetics, Part C, vol. 36, Issue 6, pp. 713-724.

Ma, C. K., J. M. Chang and D. C. Lin (2003). Input Forces Estimation Of Beam Structures by an Invers Method. Journal of Sound and Vibration, 259(2), pp. 387-407.

Maybeck P. S. (1979). Stochastic Models, Estimation and Control. Volume 1, Academic Press, Inc.

Nasir M., M. Dickinson, and D. Liepmann (2006). Measurement of Insect Flight Forces Using a MEMS Based Physical Sensor. Proceedings of
Hilton Head 2006, Solid State Sensors, Actuators and Microsystems Workshop. Hilton Head Island.

Nilsson, B., J. Nygards, U. Larsson and A. Wernersson (1999). Control of Flexible Mobile Manipulators: Positioning and Vibration Reduction Using an Eye-in-hand Range Camera. Control Engineering Practice, 7, pp. 741-751.

Woods R. J. and R. S. Fearing (2001). Flight Force Measurements for a Micromechanical Flying Insect. Proceedings of the IEEE/RSJ International Conference on Intelligent Robots and Systems, pp. 355-362 vol. 1, Hawaii, USA. 